\title{
THE KONUS-WIND CATALOG OF GAMMA-RAY BURSTS WITH KNOWN REDSHIFTS. I. BURSTS DETECTED IN THE TRIGGERED MODE.
}

\author{
A. Tsvetkova ${ }^{* a}$,D. Frederiks ${ }^{a}$, S. Golenetskii ${ }^{a}$, A. Lysenko ${ }^{a}$, P. Oleynik ${ }^{a}$, V. Pal'shin $^{b}$, \\ D. Svinkin ${ }^{a}$, M. Ulanov ${ }^{a}$, T. Cline ${ }^{c \dagger}$ K. Hurley ${ }^{d}$, R. Aptekar ${ }^{a}$ \\ ${ }^{a}$ Ioffe Institute, Politekhnicheskaya 26, St. Petersburg 194021, Russia \\ ${ }^{b}$ Vedeneeva 2-31, St. Petersburg, Russia \\ ${ }^{c}$ NASA Goddard Space Flight Center, Greenbelt, MD 20771, USA \\ ${ }^{d}$ Space Sciences Laboratory, University of California, 7 Gauss Way, Berkeley, CA 94720-7450, \\ USA \\ E-mail: tsvetkova@mail.ioffe.ru
}

\begin{abstract}
We present the results of a systematic study of 150 gamma-ray bursts (GRBs) with reliable redshift estimates detected in the triggered mode of the Konus-Wind (KW) experiment [1]. The sample covers the period from 1997 February to 2016 June and represents the largest set of cosmological GRBs studied to date over a broad energy band. We provide the burst durations, the spectral lags, the results of spectral fits with two model functions, the total energy fluences, and the peak energy fluxes, the rest-frame, isotropic-equivalent energy and peak luminosity, and the collimation-corrected values of the energetics for 32 GRBs with reasonably-constrained jet breaks. We consider the behavior of the rest-frame GRB parameters in the hardness-duration and hardness-intensity planes, and confirm the "Amati" and "Yonetoku" relations for Type II GRBs. The correction for the jet collimation does not improve these correlations for the KW sample. We discuss the influence of instrumental selection effects on the GRB parameter distributions and estimate the KW GRB detection horizon. Accounting for the instrumental bias, we estimate the KW GRB luminosity evolution, luminosity and isotropic-energy functions, and the evolution of the GRB formation rate.
\end{abstract}

7th Fermi Symposium 2017

15-20 October 2017

Garmisch-Partenkirchen, Germany

\footnotetext{
*Speaker.

${ }^{\dagger}$ Emeritus
} 


\section{The catalog sample, analyses and results}

The Konus-Wind experiment (KW) has operated since 1994 November and plays an important role in GRB studies thanks to its unique set of characteristics: the spacecraft orbit in interplanetary space that provides a stable background and continuous coverage of the full sky by two omnidirectional NaI detectors; high temporal resolution; and wide energy range of the detectors ( $\sim 20 \mathrm{keV}-20 \mathrm{MeV}$ ). The catalog sample [1] comprises $150 \mathrm{GRBs}$ with reliable redshift estimates detected by KW in the triggered mode from the beginning of the afterglow era in 1997 to the middle of 2016, and represents the largest set of cosmological GRBs studied to date over a broad energy band (compared, e.g., with [2]). Among these GRBs, twelve bursts (or 8\%) belong to the Type I (merger origin, short/hard) GRB population and the others are Type II (collapsar origin, long/soft) bursts (see [3] for the KW GRB classification details).

From the temporal and spectral analyses of the sample, we provide the burst durations $T_{100}$, $T_{90}$, and $T_{50}$, the spectral lags, and spectral fits with CPL and Band model functions. From the BEST spectral models we calculated the $10 \mathrm{keV}-10 \mathrm{MeV}$ energy fluences $(S)$ and the peak energy fluxes $\left(F_{\text {peak }}\right)$ on three time scales: $1024 \mathrm{~ms}, 64 \mathrm{~ms}$, and $(1+z) 64 \mathrm{~ms}$ (the GRB rest-frame $64 \mathrm{~ms}$ scale). Based on the GRB redshifts, which span the range $0.1 \leq z \leq 5$, we estimated the rest-frame, isotropic-equivalent energies $\left(E_{\text {iso }}\right)$ and peak luminosities $\left(L_{\text {iso }}\right)$ in the rest-frame bolometric range $1 \mathrm{keV}-(1+z) 10 \mathrm{MeV}$. For $32 \mathrm{GRBs}$ with reasonably constrained jet break times $\left(t_{\mathrm{jet}}\right)$ we provide the collimation-corrected values of the energetics.

\section{KW selection effects and GRB detection horizon}

Figure 1 shows the KW GRB distributions in the $E_{\mathrm{iso}}-z, L_{\mathrm{iso}}-z$, and $E_{\mathrm{p}, \mathrm{z}}-z$ diagrams ${ }^{1}$. The region in the $L_{\text {iso }}-z$ plane above the limit defined by the bolometric flux $F_{\lim } \sim 1 \times 10^{-6} \mathrm{erg} \mathrm{cm}^{-2} \mathrm{~s}^{-1}$ may be considered free from the selection bias. In the $E_{\text {iso }}-z$ plane, the selection-free region corresponds to the bolometric fluence $S_{\lim } \gtrsim 3 \times 10^{-6} \mathrm{erg} \mathrm{cm}^{-2}$. The detector sensitivity drops rapidly as $E_{\mathrm{p}}$ (the peak energy in $v F_{v}$ spectrum) approaches the lower boundary of the KW band, and this results in a lack of bursts below the rest-frame limit $\approx(1+z)^{2} \cdot 25 \mathrm{keV}$ in the $E_{\mathrm{p}, \mathrm{z}}-z$ plane.

Accounting for the KW trigger sensitivity to the GRB incident angle, its light-curve shape, and the shape of the energy spectrum, we estimated the KW detection horizon for each burst from the sample as a redshift $z_{\max }$, at which the GRB peak count rate in the trigger energy band $(\sim 80$ $300 \mathrm{keV})$ drops below the trigger threshold $(9 \sigma)$ on both trigger time scales (140 ms and $1 \mathrm{~s})$. The resulting values of $z_{\max }$ are shown in Figure 2. The most distant GRB horizon for the KW sample $\left(z_{\max } \approx 16.6\right)$ is reached for the ultra-luminous GRB $110918 \mathrm{~A}$ at $z=0.981$. At $z \approx 16.6$ the age of the Universe amounts to only $\sim 230 \mathrm{Myr}$, i.e. a burst which occurred close to the end of the cosmic Dark Ages could still trigger the KW detectors, and a thorough temporal and spectral analysis in a wide observer-frame energy range could be performed. Among the KW Type I GRBs the highest $z_{\max } \approx 5.3$ is for GRB $160410 \mathrm{~A}(z=1.72)$.

A common approach to estimate the GRB detection horizon is to find a redshift $z_{\max , \mathrm{L}}$, at which the limiting isotropic luminosity $L_{\text {iso, } \lim }=4 \pi D_{\mathrm{L}}^{2} \times F_{\text {lim }}$, defined by the "monolithic" $F_{\text {lim }}$ estimated for the whole sample, starts to exceed the GRB $L_{\text {iso }}$. We found that for both Type I

\footnotetext{
${ }^{1}$ We use $A_{z}$ notation for rest-frame GRB parameters.
} 
and Type II GRBs $z_{\max }$ are distributed narrowly around $z_{\max , \mathrm{L}}$ corresponding to the bolometric $F_{\text {lim }}=1 \times 10^{-6} \mathrm{erg} \mathrm{cm}^{-2} \mathrm{~s}^{-1}$.
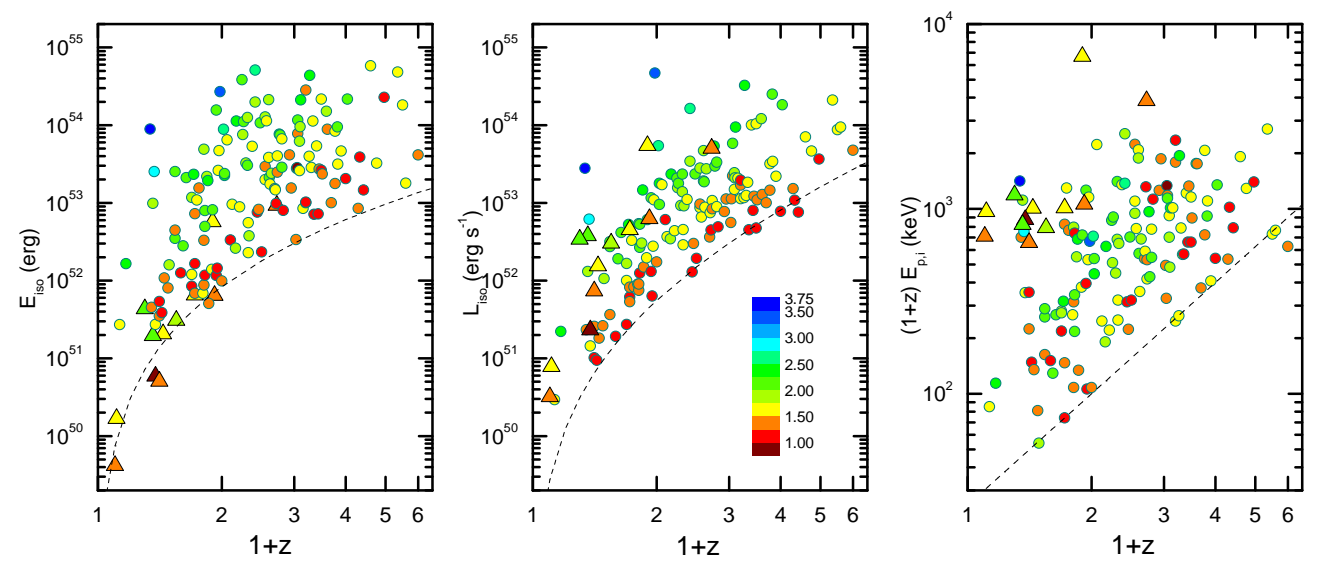

Figure 1: KW GRB $E_{\mathrm{iso}}, L_{\mathrm{iso}}$, and $E_{\mathrm{p}, \mathrm{i}, \mathrm{z}}$ vs. redshift. The color of each data point (Type I: triangles, Type II: circles) represents the log of the burst's trigger significance $(\sigma)$. The observer-frame limits are shown with dashed lines.

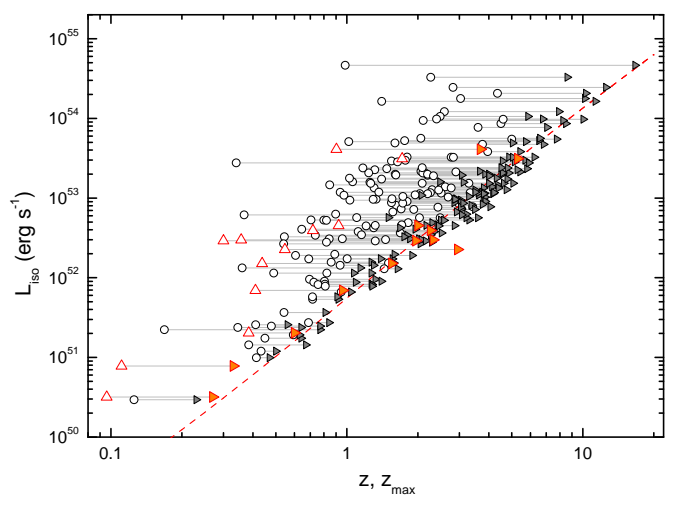

Figure 2: $\mathrm{KW}$ GRB detection horizons plotted in the $L_{\text {iso }}-z$ plane. The solid lines connect GRBs from the sample (Type I: open triangles, Type II: open circles) and their detection horizons $z_{\max }$ (filled symbols). The limiting redshift $z_{\max , \mathrm{L}}$ defined by $F_{\text {lim }}=1 \times 10^{-6} \mathrm{erg} \mathrm{cm}^{-2} \mathrm{~s}^{-1}$ is shown by the dashed line.

\section{GRB Luminosity and Isotropic-energy functions, GRB Formation Rate}

To estimate the GRB luminosity function (LF), isotropic energy release function (EF), and the cosmic GRB formation rate (GRBFR) for the KW Type II bursts we used the non-parametric Lynden-Bell $C^{-}$method ([4]) further advanced by [5] (the EP method); the details of our calculations are described in [1]. Applying the EP to to the $z-L_{\text {iso }}$ plane and using the functional form of the evolution $g(z)=(1+z)^{\delta}$, we found the luminosity evolution significance $\tau_{0} \sim 1.7 \sigma$, and 
estimated the luminosity evolution index $\delta_{L}=1.7_{-0.9}^{+0.9}$. Applying the same method to the $z-E_{\text {iso }}$ plane, $\delta_{E}=1.1_{-0.7}^{+1.5}\left(\tau_{0} \sim 1.6 \sigma\right)$. Thus, the estimated $E_{\text {iso }}$ and $L_{\text {iso }}$ evolutions are comparable.

Then, using the $C^{-}$method, we obtained evolution-free cumulative LF and EF, $\psi\left(L^{\prime}\right)$ and $\psi\left(E^{\prime}\right)$ (Figure 3), where $L^{\prime}=L_{\text {iso }} /(1+z)^{\delta_{L}}$ and $E^{\prime}=E_{\text {iso }} /(1+z)^{\delta_{E}}$, and fitted the distributions with a broken power-law (BPL) function and with the exponentially-cutoff PL (CPL). The derived BPL slopes of LF and EF are close to each other, both for the $\operatorname{dim}\left(\alpha_{1} \sim-0.5\right)$ and bright $\left(\alpha_{2} \sim-1\right)$ segments; also, these indices are roughly consistent with the LF and EF slopes obtained in [6] and [7]. When compared to BPL, the CPL fit to $\psi\left(L^{\prime}\right)$ results in a considerably worse quality. Conversely, the cutoff PL fits $\psi\left(E^{\prime}\right)$ better $\left(\Delta \chi^{2} \sim 5.5\right)$, with the PL slope $\sim-0.35$ and the cutoff energy $E_{\text {cut }}^{\prime} \sim 2.3 \times 10^{54} \mathrm{erg}$. The existence of a sharp cutoff of the GRB isotropic energy distribution around $\sim 1-3 \times 10^{54}$ erg was suggested recently by [8]. We also estimated the LF and EF not accounting for the evolution, and found them very similar in shape to $\psi\left(L^{\prime}\right)$ and $\psi\left(E^{\prime}\right)$.

The GRBFR (Figure 3), estimated using the EP method from the evolution-corrected $z-L^{\prime}$ distribution, exceeds the star formation rate (SFR) at $z<1$ and nearly traces the SFR at higher redshifts; the same behavior is noted for the GRBFRs estimated using both the evolution-corrected $z-E^{\prime}$ and the non-corrected $z-E_{\text {iso }}$ distributions. The low- $z$ GRBFR excess over SFR is in agreement with the results reported in [9] and [10]. Meanwhile, the only GRBFR that traces the SFR in the whole KW GRB redshift range is the one derived from the $z-L_{\text {iso }}$ distribution (i.e. not accounting for the luminosity evolution).
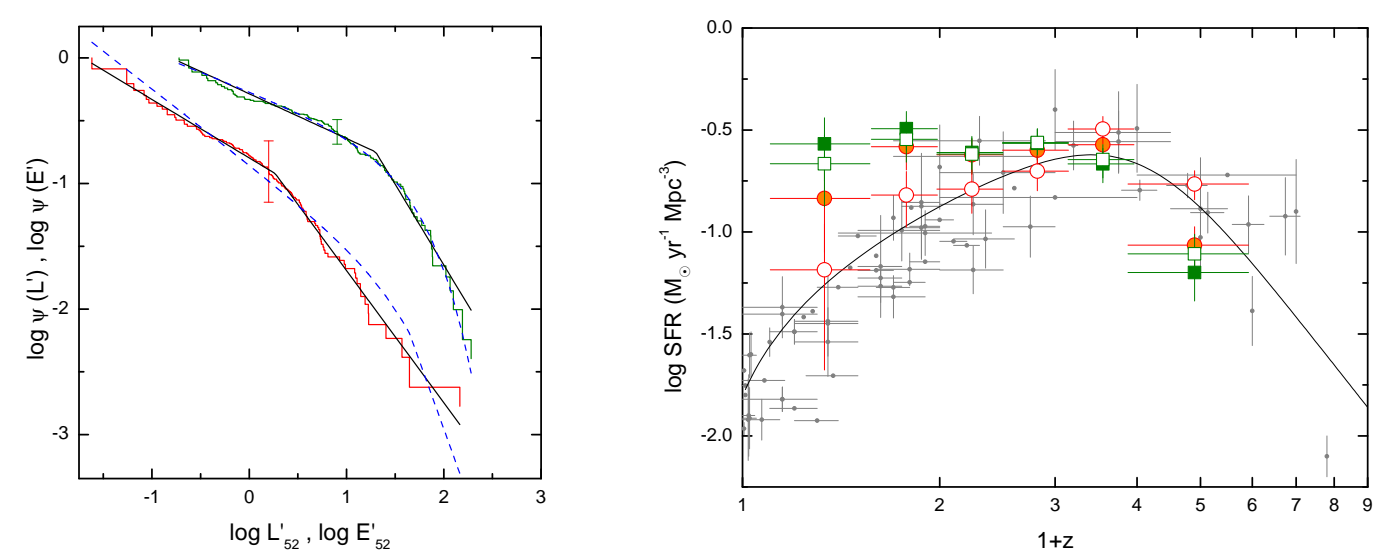

Figure 3: Left: cumulative LF (red stepped graph) and EF (green stepped graph) estimated accounting for the luminosity and energy evolutions; the solid and dashed lines show the best BPL and CPL fits, respectively. Right: GRBFR calculated using four datasets: $z-L_{\text {iso }}$ (no luminosity evolution, red open circles), $z-L^{\prime}$ ( $\delta_{L}=1.7$, red filled circles), $z-E_{\text {iso }}$ (no energy evolution, green open squares), and $z-E^{\prime}$ ( $\delta_{E}=1.1$, green filled squares). The gray points and the solid line show the SFR data from the literature (for references see [1]). The GRBFR points have been shifted arbitrarily to match the SFR at $(1+z) \sim 3.5$.

\section{Hardness-intesity correlations}

We tested the KW GRB characteristics against $E_{\mathrm{p}}-S$ and $E_{\mathrm{p}}-F_{\text {peak }}$ correlations in the observer 
frame, and $E_{\mathrm{p}, \mathrm{z}}-E_{\mathrm{iso}}$ ("Amati", [11]) and $E_{\mathrm{p}, \mathrm{z}}-L_{\mathrm{iso}}$ ("Yonetoku", [6]) correlations in the rest frame. For the subsamples of Type I and Type II KW GRBs both the Amati and Yonetoku correlations improve considerably when moving from the observer frame to the GRB rest frame, with only marginal changes in the slopes. The derived slopes of the Amati and Yonetoku relations for Type II GRBs are $\sim 0.47$ and $\sim 0.49$, respectively. These values are in agreement with [11], [6]. For $30 \mathrm{KW}$ Type II GRBs with reliable $t_{\text {jet }}$, accounting for the jet collimation neither improves the significance of the correlations nor reduces the dispersion of the points around the best-fit relations. The slopes we obtained for the collimated Amati and Yonetoku relations are steeper compared to those of the non-collimated versions.

The lower boundaries of both the Amati and Yonetoku relations (Figure 4) are defined by GRBs with moderate-to-high detection significance, so the instrumental biases do not affect the correlations from this edge of the distributions. Meanwhile, all outliers in the relations lie above the upper boundaries of the $90 \%$ prediction intervals (PIs) of the relations. Since these bursts were detected at lower significance, with the increased number of GRB redshift observations, one could expect a "smear" of the correlations due to more hard-spectrum/less-energetic GRB detections. Thus, using the KW sample, we confirm a finding of [12] that the lower right boundary of the Amati correlation (the lack of luminous soft GRBs) is an intrinsic GRB property, while the top left boundary may be due to selection effects. This conclusion may also be extended to the Yonetoku correlation.

The $E_{\mathrm{p}, \mathrm{z}}-E_{\mathrm{iso}}$ and $E_{\mathrm{p}, \mathrm{z}}-L_{\text {iso }}$ correlations for 12 Type I bursts are less significant when compared to those for Type II GRBs, and they are characterized by less steep slopes. Meanwhile, the restframe $E_{\mathrm{p}}$ of Type I GRBs shows only a weak (if any) dependence on the burst energy below $E_{\text {iso }} \sim$ $10^{52} \mathrm{erg}$ (Figure 4), and the same is true for the $E_{\mathrm{p}, \mathrm{z}}-L_{\text {iso }}$ relation at $L_{\text {iso }} \lesssim 5 \times 10^{52} \mathrm{erg} \mathrm{s}^{-1}$. Above these limits the slopes of both relations for Type I GRBs are similar to those for Type II GRBs. As one can see from the Figure, all KW Type I bursts are hard-spectrum/low-isotropic-energy outliers in the Amati relation for Type II GRBs. In the $E_{\mathrm{p}, \mathrm{z}}-L_{\text {iso }}$ plane this pattern is less distinct; at luminosities above $L_{\text {iso }} \sim 10^{52} \mathrm{erg} \mathrm{s}^{-1}$ the Type I bursts nearly follow the upper boundary of the Type II GRB Yonetoku relation.

\section{Conclusions}

We hope this catalog will encourage further investigations of GRB physical properties and will contribute to other related studies. Plots of the GRB light curves and spectral fits can be found at the Ioffe Web site ${ }^{2}$. This work was supported by RSF (grant 17-12-01378).

\section{References}

[1] A. Tsvetkova, D. Frederiks, S. Golenetskii, A. Lysenko, P. Oleynik, V. Pal'shin et al., The Konus-Wind catalog of gamma-ray bursts with known redshifts. I. Bursts detected in the triggered mode, ArXiv e-prints (Oct., 2017) , [1710.08746].

[2] D. Gruber, J. Greiner, A. von Kienlin, A. Rau, M. S. Briggs, V. Connaughton et al., Rest-frame properties of 32 gamma-ray bursts observed by the Fermi Gamma-ray Burst Monitor, A\&A 531 (July, 2011) A20, [1104.5495].

\footnotetext{
${ }^{2}$ http://www.ioffe.ru/LEA/zGRBs/triggered/
} 

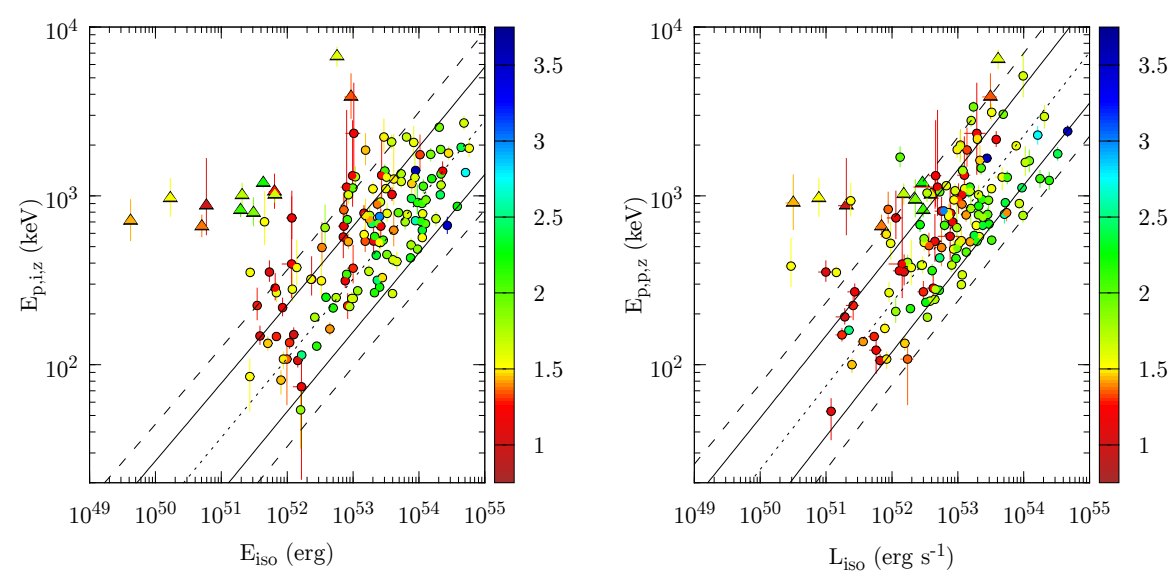

Figure 4: Rest-frame energetics in the $E_{\mathrm{iso}}-E_{\mathrm{p}, \mathrm{z}}$ (left) and $L_{\mathrm{iso}}-E_{\mathrm{p}, \mathrm{z}}$ (right) planes. The color of each data point (Type I: triangles, Type II: circles) represents the log of the burst's trigger significance. The "Amati" and "Yonetoku" relations for Type II GRBs are plotted with dotted lines; the solid and dashed lines show their $68 \%$ and $90 \%$ PI's, respectively.

[3] D. S. Svinkin, D. D. Frederiks, R. L. Aptekar, S. V. Golenetskii, V. D. Pal'shin, P. P. Oleynik et al., The Second Konus-Wind Catalog of Short Gamma-Ray Bursts, ApJS 224 (May, 2016) 10, [1603.06832].

[4] D. Lynden-Bell, A method of allowing for known observational selection in small samples applied to 3CR quasars, MNRAS 155 (1971) 95.

[5] B. Efron and V. Petrosian, A simple test of independence for truncated data with applications to redshift surveys, ApJ 399 (Nov., 1992) 345-352.

[6] D. Yonetoku, T. Murakami, T. Nakamura, R. Yamazaki, A. K. Inoue and K. Ioka, Gamma-Ray Burst Formation Rate Inferred from the Spectral Peak Energy-Peak Luminosity Relation, ApJ 609 (July, 2004) 935-951, [arXiv: astro-ph/0309217].

[7] S.-W. Wu, D. Xu, F.-W. Zhang and D.-M. Wei, Gamma-ray bursts: the isotropic-equivalent-energy function and the cosmic formation rate, MNRAS 423 (July, 2012) 2627-2632, [1112 . 2029].

[8] J.-L. Atteia, V. Heussaff, J.-P. Dezalay, A. Klotz, D. Turpin, A. E. Tsvetkova et al., The Maximum Isotropic Energy of Gamma-ray Bursts, ApJ 837 (Mar., 2017) 119, [1702.02961].

[9] H. Yu, F. Y. Wang, Z. G. Dai and K. S. Cheng, An Unexpectedly Low-redshift Excess of Swift Gamma-ray Burst Rate, ApJS 218 (May, 2015) 13, [1504.01812].

[10] V. Petrosian, E. Kitanidis and D. Kocevski, Cosmological Evolution of Long Gamma-Ray Bursts and the Star Formation Rate, ApJ 806 (June, 2015) 44, [1504.01414].

[11] L. Amati, F. Frontera, M. Tavani et al., Intrinsic spectra and energetics of BeppoSAX Gamma-Ray Bursts with known redshifts, $A \& A 390$ (2002) 81.

[12] V. Heussaff, J.-L. Atteia and Y. Zolnierowski, The $E_{\text {peak }}-E_{\text {iso }}$ relation revisited with Fermi GRBs. Resolving a long-standing debate?, A\&A 557 (Sept., 2013) A100, [1306.1757]. 